\title{
Cervical Spinal Epidural Abscess Following Acupuncture: Successful Treatment with Antibiotics
}

\author{
Shogo Yazawa, Takekazu Ohi, Sei-ichiro Sugrmoto, Sei-ichi Satoh and Shigeru Matsukura
}

\begin{abstract}
A 67-year-old man with poorly controlled diabetes mellitus (DM) had acupuncture several times a month for chronic shoulder muscle stiffness. A few days after acupuncture in the posterior nuchal region, a low-grade fever and backache developed, and subacutely progressed. Finally he complained of gait disturbance, and then respiratory distress appeared. Magnetic resonance imaging (MRI) demonstrated high cervical epidural abscess with massive soft tissue inflammation and vertebral osteomyelitis. Conservative treatment with antibiotics was effective and it was well documented by following serial MRIs. This case suggested that needle acupuncture should be avoided for immunocompromised subjects such as patients with poorly controlled DM.
\end{abstract} (Internal Medicine 37: 161-165, 1998)

Key words: bacterial infection, conservative therapy, spinal magnetic resonance imaging (MRI)

\section{Introduction}

Spinal epidural abscess is an uncommon infectious disorder in the spinal epidural space. Moreover, it occurs even less frequently at the cervical than at the thoracic and lumbar spine (1). Although earlier diagnosis leads to a better prognosis, at times it has been misdiagnosed $(1,2)$. Recently, magnetic resonance imaging (MRI) has been found to be very useful for diagnosing spinal epidural abscess and for detecting associated abnormalities of the spinal cord, vertebral bodies, intervertebral disc, and paraspinal soft tissue (2-5). As for its pathogen, Staphylococcus aureus (S. aureus) is the most common causative organism $(1,3,6)$, and the appropriate invasive procedure should be always performed after diagnosis (1).

Herein, we describe a patient with a cervical spinal epidural abscess that developed following needle acupuncture in the posterior nuchal region, and in whom conservative therapy was effective.

\section{Case Report}

A 67-year-old, right-handed Japanese man had suffered from diabetes mellitus (DM) for about 15 years, and had been treated with oral antidiabetic medications, but it was poorly controlled. In addition, he usually received needle acupuncture in the nuchal and lumbar regions once a week for his intractable shoulder stiffness and lumbar pain. Low-grade fever, backache, and posterior neck pain subacutely developed on September 20, 1992. Four days later, he could not stand up by himself. On September 28, thereafter, he could not walk; and so he visited a local hospital with his family. At that time, physical examination revealed high fever and marked nuchal rigidity. As polymorphonuclear pleocytosis $\left(147 / \mathrm{mm}^{3}\right)$ and an increased protein level $(295 \mathrm{mg} / \mathrm{dl})$ in the cerebrospinal fluid (CSF) were observed, he was diagnosed as having acute bacterial meningitis. In spite of parenteral antibiotic administration (cefotaxime, $4 \mathrm{~g} / \mathrm{day}$, DIV), the muscular weakness extended to his upper limbs. Two weeks later after the onset, he was transferred to our hospital for neurological care.

On admission, he had low-grade fever and marked inflammatory swelling and severe tenderness in the posterior neck. Neurological examination showed marked nuchal stiffness, spastic quadriplegia, generalized hyperreflexia with bilateral extensor plantar responses, and sensory impairment of all modalities below $\mathrm{C} 3$. His respiratory function was severely disturbed (forced vital capacity, $700 \mathrm{ml}$; tidal volume, $300 \mathrm{ml}$ ).

Urinalysis showed moderate glycosuria, proteinuria, and ketonuria. Hematological examination and blood chemistry revealed increased white blood cell (WBC) $\left(11,000 / \mathrm{mm}^{3}\right)$ with neutrophilia, mild hypoalbuminemia $(2.59 \mathrm{~g} / \mathrm{dl})$, and an increased $\gamma$-globulin level (24\%). Fasting blood sugar was 260 $\mathrm{mg} / \mathrm{dl}$. There were pronounced inflammatory reactions [erythrocyte sedimentation rate (ESR), $70 \mathrm{~mm} / \mathrm{h}$; C-reactive protein (CRP), $13.7 \mathrm{mg} / \mathrm{dl})$ ]. Anti-human T-cell leukemia virus type-I

From the Division of Neurology, Department of Internal Medicine, Miyazaki Medical College, Miyazaki

Received for publication April 28, 1997; Accepted for publication October 17, 1997

Reprint requests should be addressed to Dr. Takekazu Ohi, the Division of Neurology, Department of Internal Medicine, Miyazaki Medical College, 5200 Kihara, Kiyotake, Miyazaki 889-1601 
(HTLV-I) antibody was negative in both plasma and CSF. Examination of the CSF disclosed a markedly elevated protein level $(1,230 \mathrm{mg} / \mathrm{dl})$, mild polymorphonuclear pleocytosis (15 cells $\left./ \mathrm{mm}^{3}\right)$, and a significantly diminished glucose level (17 $\mathrm{mg} / \mathrm{dl})$; in contrast, the chloride level was normal. Repeated cultures of blood and CSF were negative, throughout the whole clinical course.

A T2-weighted MRI of cervical spine on the 21st day from the onset showed an epidural mass with an increased highintensity signal around $\mathrm{C} 1$ to $\mathrm{C} 2$, and a high intensity signal area within the swollen upper spinal cord (Fig. 1). The epidural mass appeared as a low intensity area on T1-weighted image displacing the spinal cord posteriorly at $\mathrm{C} 1$ to $\mathrm{C} 2$, and was delineated by peripheral enhancement with gadolinium-DTPA. In addi- tion, massive subcutaneous inflammation (cellulitis) of posterior nuchal and paraspinal areas, discitis at C5 to C6, thickening of anterior and posterior longitudinal ligaments and small abscesses surrounding the high cervical and thoracic regions were shown. Osteomyelitis at $\mathrm{C} 1$ and $\mathrm{C} 2$ vertebra were also evident (Fig. 1). This epidural abscess, compressing the spinal cord, accounted for the quadriparesis and respiratory insufficiency observed in the patient. The swelling of the spinal cord was likely due to compression, however, the possibility of myelitis of the spinal cord was not negligible.

After the transfer to our hospital, sensory impairment and respiratory distress of the patient subacutely worsened within a week (Fig. 2). Neither surgical decompression nor drainage was chosen by neurosurgeons because of massive subcutane-

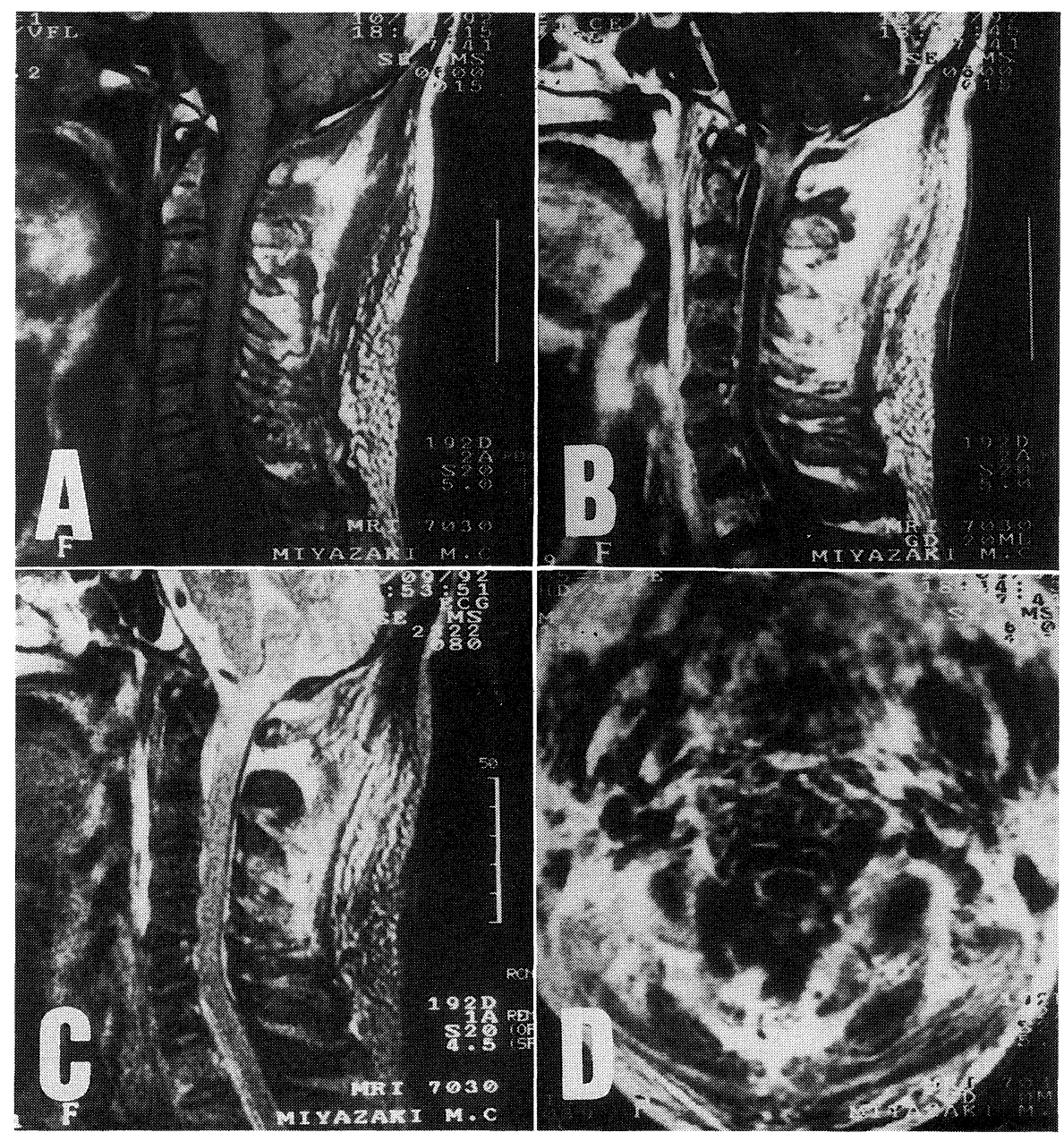

Figure 1. Cervical MR imaging on the 21st day from the onset. Abscess is seen at C1C2 anterior epidural space, it is low intensity on T1WI (A, TR/TE: 600/15), and is clearly delineated by peripheral enhancement of Gd-DTPA (B, TR/TE: 600/15), and T2WI (C, TR/ TE: 2,322/80) MRI shows a high intensity signal at that area. Massive subcutaneous inflammation at the posterior nuchal region, osteomyelitis of $\mathrm{C} 1$ and $\mathrm{C} 2$ vertebra, and pachy distributed soft tissue inflammation are observed $(\mathrm{C})$. Anterior and posterior longitudinal ligaments are also involved (B). Compression of the upper cervical spinal cord by the epidural abscess was seen in the axial view (D, TR/TE: 600/15, with Gd-DTPA enhancement). 


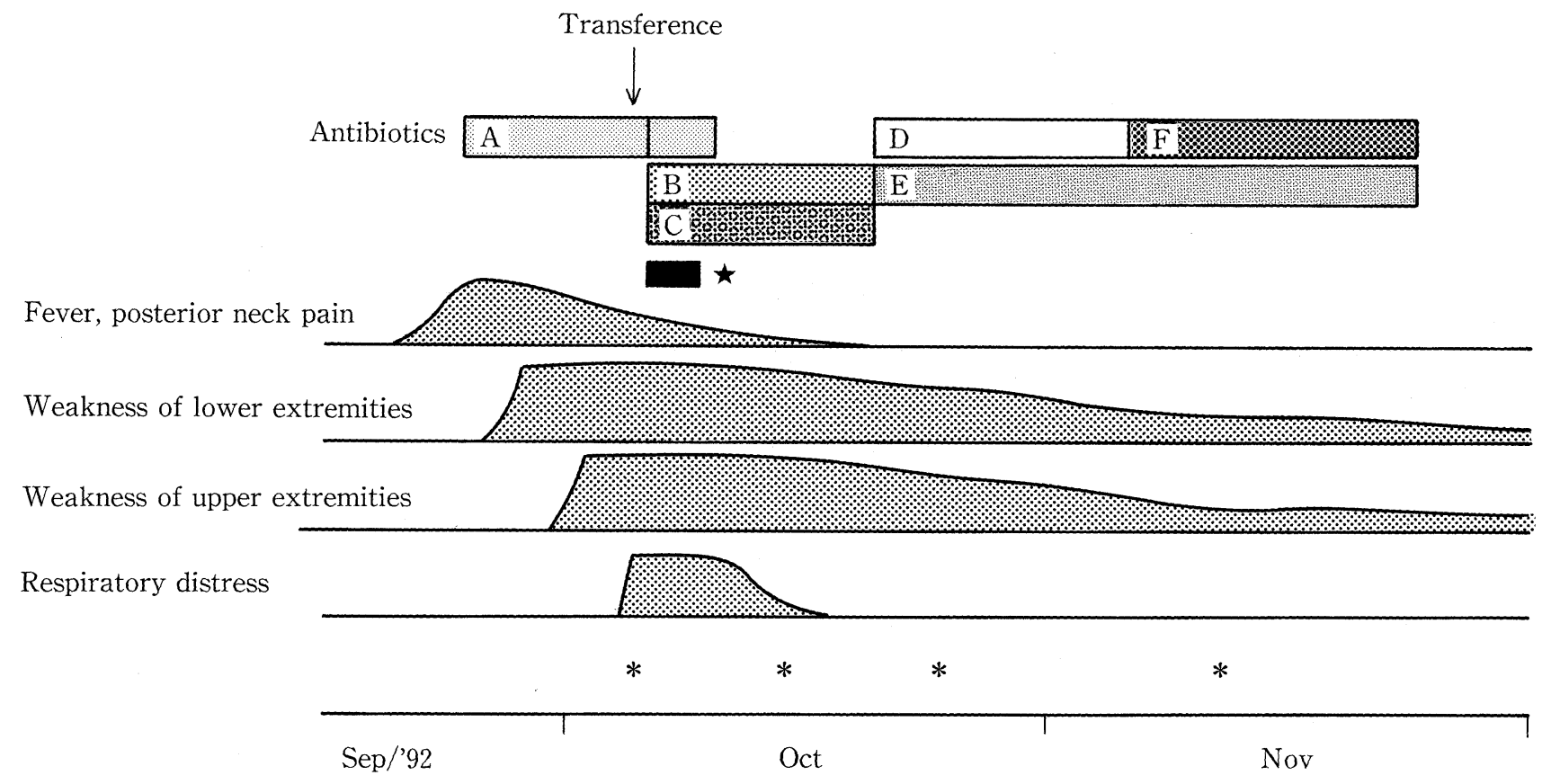

A: CTX, 4 g/day, B: TOB, 80 mg/day, C: PIPC, 6 g/day, D: IPM/CS, 2 g/day, E: ABPC, 4 g/day,

$\mathrm{F}: \mathrm{CAZ}, 1 \mathrm{~g} /$ day, $\star$ dexamethasone, $16 \mathrm{mg}$

Figure 2. Clinical course of this patient. Neurological signs were gradually improved after starting intensive administration of antibiotics. Cervical MRI was serially examined at the point indicated by asterisk (*). CTX: cefotaxmine, TOB: tobramycin, PIPC: piperacillin, IPM/CS: imipenem/cilastatin, ABPC: aminobensilpenicillin, CAZ: ceftazizim, DEX: dexamethasone.

ous inflammation in the posterior nuchal region. Although no specific pathogen was identified, $\mathbf{S}$. aureus was suspected from the clinical standpoint. Thus, we chose the combination of ampicillinase-resistant penicillin and a third-generation cephem were added to the cefotaxime (CTX) treatment, $4 \mathrm{~g} /$ day, that had already begun beforehand. Afterwards, clindamycin (CLDM), imipenem (IPM/CS), aminobensilpenicillin (ABPC) and ceftazizim (CAZ) were also used. For reduction of the edema around the compressed spinal cord, intravenous dexamethasone, $16 \mathrm{mg} / \mathrm{day}$, was given for the first 3 days; and the dosage was then tapered. Following the start of these treatments, the muscle strength of his upper extremities improved and the respiratory insufficiency lessened in a few weeks, and muscle strength of bilateral lower extremities and sensory impairment were gradually restored over five months. The high blood glucose level of the patient was controlled by continuous rapid-acting insulin infusion given intravenously.

Spinal MRIs were serially studied as shown in Fig. 3. Spinal epidural abscesses at $\mathrm{C} 1$ to $\mathrm{C} 2$, discitis at $\mathrm{C} 5$ to $\mathrm{C} 6$ intervertebral space and subcutaneous soft tissue inflammation were ameliorated. However, the increased high intensity area within the cervical spinal cord on T2WI persisted, which might imply intraspinal gliosis after compression.

\section{Discussion}

There are some points to discuss in the present patient; 1) misdiagnosis as a simple meningitis at the primary local hospital in spite of evidence of neurological manifestations, 2) etiology of the abscess and 3) good outcome of the conservative treatment.

Spinal epidural abscess is a relatively rare disorder $(0.2-1.2$ per 10,000 of all hospital admissions) (1). Moreover, cervical lesion is the least frequently involved, which is approximately $15.6 \%$ of all spinal epidural abscesses (3). Although early diagnosis is certainly crucial for successful treatment, spinal epidural abscess was correctly suspected initially in only 20 $25 \%$ of cases $(1,7)$. The clinical course of spinal epidural abscess has been classified into four stages: spinal ache, root pain, weakness, and paralysis (8). Unfortunately our patient was diagnosed at the final stage. The major mis-diagnoses were extruded disk, spinal tumor, vertebral osteomyelitis, musculoskeletal arthritis, meningitis, and transverse myelitis (1). The present case was also initially suspected by a general internist as meningitis until the appearance of paraparesis. General internists should consider the possibility of spinal epidural abscess when patients show spinal cord signs accompanied by high fever. In addition, MRI has a great advantage in diagnosis and follow-up of such an abscess $(2,4,5)$. It should be performed promptly in any patient with clinical features suggesting spinal epidural abscess since early surgical and medical treatment can yield a favorable outcome.

The major sources of infection include osteomyelitis, bacteremia, and postoperative infection. The most common 

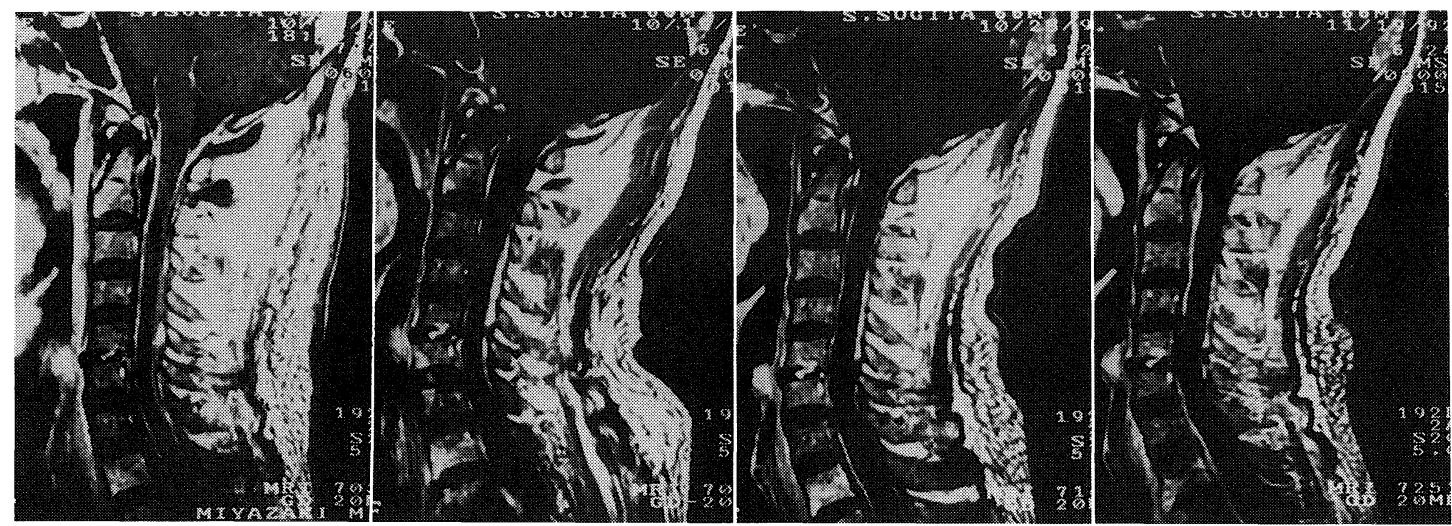

day 21

day 31

day 40

day 61
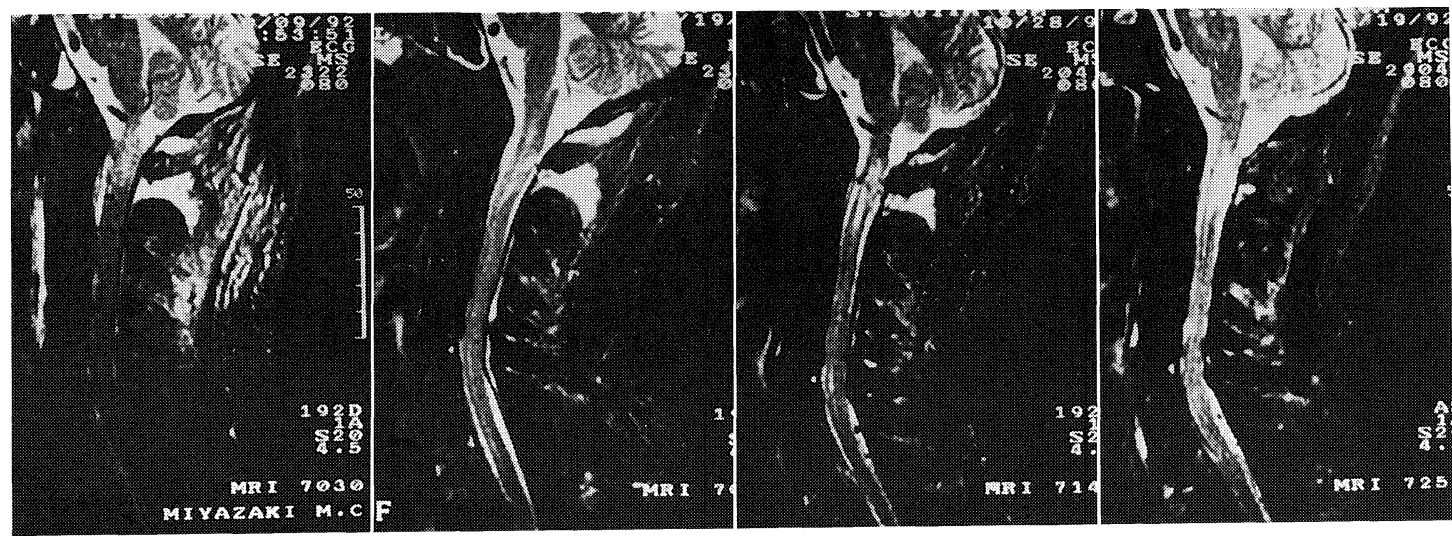

(MMC \# 155211-7)

Figure 3. Serial changes of follow-up MRIs. Gd-DTPA-enhanced T1WI images (TR/TE: 600/15, upper) and T2WI images (TR/TE: 2,322/80, lower) are serially shown. By antibiotic treatment, the epidural abscess, osteomyelitis and subcutaneous inflammation were improved. The high intensity signal on T2WI of the cervical intramedullary region still remained even when the patient had clinically recovered (day 61).

infectious route of cervical spinal epidural abscess is by hematogenous spread and is usually associated with a remote source of infection involving the arm, neck, or head (6). However, the cervical epidural space is the least hematogenous infectious area among the whole spine, because of its narrower anteroposterior diameter $(0.1 \mathrm{~cm})$ and poorer venous plexus in cervical epidural space as compared with those of thoracic spine $(0.5 \sim 0.75 \mathrm{~cm})(7,9)$. In this case, there was no preceding infection; but the patient did have a long history of undergoing acupuncture in the posterior nuchal region. Needle acupuncture is a very popular traditional treatment, mainly in East Asian countries, for relief of intractable chronic pain or other chronically ill conditions such as headache, numbness and weakness. $\mathrm{S}$. aureus infections in the psoas muscles and lumbar epidural space caused by needle acupuncture have been previously reported $(10,11)$. Although we did not detect any specific organisms in the present patient, S. aureus, the most common bacterium in human skin, is also the most frequent causative organism of spinal epidural abscess $(1,3,6,12)$. In addition, well-known conditions predisposing to $S$. aureus infection are
DM, alcohol abuse, intravenous drug abuse, trauma, and immunosuppression (12). We suspect that acupuncture, by initiating an infectious process caused by S. aureus, may have led to the cervical epidural abscess in this patient in poorly controlled DM.

The choice of the treatment of this disorder has been debated as to whether surgical procedure should be required for all cases or not (13). Fortunately the infection in our patient responded to antibiotics. The following clinical conditions are essential prior to non-operative treatment: identification of the pathogenic organism, neurologically stable condition, easy access to MRI or computed tomography (CT), and immediate availability of a neurosurgeon, if warranted (14). Once medical treatment is chosen, daily close neurological evaluation of the patient should be performed to monitor the severity as recommended by Baker et al (13).

\section{References}

1) Baker AS, Ojemann RG, Swartz MN, Richardson EP Jr. Spinal epidural 


\section{Epidural Abscess Caused by Acupuncture}

abscess. N Engl J Med 293: 463, 1975.

2) Kricun R, Shoemaker EI, Chvaned GI, et al. Epidural abscess of the cervical spine: MR findings in five cases. Am J Roentgenol 158: 1145, 1992.

3) Darouiche RO, Hamill RJ, Greenberg SB, Weather SW, Musher DM. Bacterial spinal epidural abscess. Review of 43 cases and literature survey. Medicine (Baltimore) 71: 369, 1992.

4) Friedmand DP, Hills JR. Cervical epidural spinal infection: MR imaging characteristics. Am J Roentgenol 163: 699, 1994.

5) Sadato N, Numaguchi $Y$, Rigamonti D, et al. Spinal epidural abscess with gadolinium-enhanced MRI: serial follow up studies and clinical correlations. Neuroradiology 36: 44, 1994.

6) Danner RL, Hartman BJ. Update of spinal epidural abscess: 35 cases and review of the literature. Rev Infect Dis 9: 265, 1987.

7) Lasker BR, Harter DH. Cervical epidural abscess. Neurology 37: 1747, 1987.

8) Heusner AP. Nontuberculous spinal epidural infections. N Engl J Med
239: 845, 1948.

9) Bouchez B, Arnott G, Delfosse JM. Acute spinal epidural abscess. J Neurol 231: 343, 1985.

10) Garcia AA, Venkataramani A. Bilateral psoas abscesses following apuncture. West J Med 161: 90, 1994 (letter).

11) Hadden WA, Swanson AJ. Spinal infection caused by acupuncture mimicking a prolapsed intervertebral disc. A case report. J Bone Joint Surg [Am] 64: 624, 1982.

12) Schafer F, Mattle HP. Neurologic manifestations of Staphylococcus aureus infections. Analysis of 43 patients. Schweiz Arch Neurol Psychiatr 145: 25, 1994.

13) Baker AS, Ojemann RG, Baker RA. To decompress or not to decompress - spinal epidural abscess. Clin Infect Dis 15: 28, 1992 (editorial; comment).

14) Hanigan WC, Asner NG, Elwood PW. Magnetic resonance imaging and the non-operative treatment of spinal epidural abscess. Surg Neurol 34: 408, 1990. 